\title{
On the Capacity-Achieving Input Covariance for Multicarrier Communications over Doubly Selective Channels
}

\author{
Georg Tauböck and Franz Hlawatsch \\ Institute of Communications and Radio-Frequency Engineering, Vienna University of Technology \\ Gusshausstrasse 25/389, A-1040 Vienna, Austria \\ gtauboec@nt.tuwien.ac.at
}

\begin{abstract}
We consider pulse-shaping multicarrier (MC) communications over a (possibly rapidly varying) doubly selective channel with uncorrelated scattering. Assuming transmission free of intersymbol interference (while intercarrier interference is not constrained), we show that the statistical properties of the MC system are invariant under cyclic shifts of the subcarriers. This invariance is then used to derive structural properties of the capacity-achieving input covariance function. We show that capacity can be achieved by transmit symbols that are uncorrelated over time and cyclically stationary with respect to the frequency (subcarrier) index. We also show that capacityachieving precoding can be equivalently realized by a suitable adaptation of the transmit pulse. For classical OFDM and transmission over a WSSUS channel, precoding can be completely avoided if pulse-shaping MC transmission is used instead.
\end{abstract}

\section{INTRODUCTION}

Orthogonal frequency division multiplexing (OFDM) and its generalization, pulse-shaping multicarrier (MC) modulation, are attractive schemes for broadband wireless communications. When they operate over doubly selective fading channels, intersymbol interference (ISI) and intercarrier interference (ICI) may occur. In $[1,2]$, the system capacity of pulse-shaping MC systems over doubly selective channels has been investigated assuming that channel state information (including ISI/ICI) is available at the receiver but not at the transmitter.

In this paper, we additionally assume statistical channel knowledge at the transmitter. The covariance of the channel input process can then be adapted to the channel statistics by linear precoding [3-5]. Adopting an information-theoretical viewpoint, a precoder can be calculated from the capacityachieving input covariance (CAIC). In contrast to [3-5], this precoder will then be optimum in the sense of an informationtheoretic performance criterium (system capacity).

Here, we shall determine structural properties of the CAIC and of the associated optimum precoder for pulse-shaping MC communications over a doubly selective fading channel. The channel statistics are assumed to conform to the uncorrelated scattering (US) model, which is less restrictive than the usual wide-sense stationary uncorrelated scattering (WSSUS) model [6]. Our results are based on a statistical analysis of the system channel comprising the MC modulator, the US physical channel, and the MC demodulator. We will demonstrate strong symmetries in the statistics of this system channel that are dif- ferent from the symmetries that have been recently exploited for characterizing the CAIC for certain classes of multipleinput/multiple-output (MIMO) channels [7-11].

This paper is organized as follows. In Section II, the system model is presented. A statistical analysis is performed in Section III, leading to a derivation of structural properties of the CAIC in Section IV. In Section V, we show that optimum precoding can be realized by an adaptation of the transmit pulse that becomes time-invariant for a WSSUS channel.

\section{SySTEM MODEL}

\section{A. Uncorrelated-Scattering Doubly Selective Channel}

We consider a discrete-time doubly selective baseband channel $\mathbb{H}$ with impulse response $h(n, m)$. The channel output is

$r(n)=(\mathbb{H} s)(n)+z(n)=\sum_{m=-\infty}^{\infty} h(n, m) s(n-m)+z(n)$,

where $s(n)$ and $z(n)$ denote the channel input and the noise, respectively, and $n, m \in \mathbb{Z}$. We also have

$$
(\mathbb{H} s)(n)=\sum_{m=-\infty}^{\infty} \int_{0}^{1} S_{\mathbb{H}}(m, \xi) s(n-m) e^{j 2 \pi \xi n} d \xi,
$$

with the channel's spreading function [6]

$$
S_{\mathbb{H}}(m, \xi) \triangleq \sum_{n=-\infty}^{\infty} h(n, m) e^{-j 2 \pi \xi n}
$$

The impulse response samples $h(n, m)$ are assumed zeromean, rotationally invariant (or proper), jointly complex Gaussian random variables satisfying the US property [6]

$$
\mathrm{E}\left\{h(n, m) h^{*}\left(n^{\prime}, m^{\prime}\right)\right\}=R_{\mathbb{H}}\left(n, n^{\prime}, m\right) \delta\left(m-m^{\prime}\right) .
$$

Here, E denotes expectation, $R_{\mathbb{H}}\left(n, n^{\prime}, m\right)$ is a temporal channel correlation function, and $\delta(\cdot)$ is the unit sample. With (3), the US property can be expressed as

$$
\mathrm{E}\left\{S_{\mathbb{H}}(m, \xi) S_{\mathbb{H}}^{*}\left(m^{\prime}, \xi^{\prime}\right)\right\}=B_{\mathbb{H}}\left(m, \xi, \xi^{\prime}\right) \delta\left(m-m^{\prime}\right),
$$

where $B_{\mathbb{H}}\left(m, \xi, \xi^{\prime}\right)$ is related to $R_{\mathbb{H}}\left(n, n^{\prime}, m\right)$ through a double Fourier transform. For a WSSUS channel, $B_{\mathbb{H}}\left(m, \xi, \xi^{\prime}\right)=$ $\tilde{B}_{\mathbb{H}}(m, \xi) \delta^{(1)}\left(\xi-\xi^{\prime}\right)$, where $\tilde{B}_{\mathbb{H}}(m, \xi)$ is the channel's scattering function [6] and $\delta^{(1)}(\cdot)$ is the Dirac impulse periodized with period 1 . 
Finally, the noise $z(n)$ is a zero-mean, stationary, white, rotationally invariant, complex Gaussian random process with variance $\sigma_{z}^{2}$; it is independent of $h(n, m)$ and $s(n)$.

\section{B. Pulse-Shaping Multicarrier Modulation}

We consider a pulse-shaping MC system with $K$ subcarriers and symbol period $N$ [12]. The modulator produces the transmit signal

$$
s(n)=\sum_{l=-\infty}^{\infty} \sum_{k=0}^{K-1} a_{l, k} g_{l, k}(n),
$$

where $a_{l, k}$ with $l \in \mathbb{Z}, k \in\{0, \ldots, K-1\}$ denotes the data symbols and

$$
g_{l, k}(n) \triangleq g(n-l N) e^{j 2 \pi \frac{k}{K}(n-l N)}
$$

is a time-frequency shifted version of a transmit pulse $g(n)$. The demodulator computes the inner products of the received signal $r(n)$ with time-frequency shifted versions $\gamma_{l, k}(n) \triangleq$ $\gamma(n-l N) e^{j 2 \pi \frac{k}{K}(n-l N)}$ of a receive pulse $\gamma(n)$, i.e.,

$$
x_{l, k}=\left\langle r, \gamma_{l, k}\right\rangle=\sum_{n=-\infty}^{\infty} r(n) \gamma_{l, k}^{*}(n) .
$$

We note that if $g(n)$ is 1 on $[0, N-1]$ and 0 otherwise and $\gamma(n)$ is 1 on $[N-K, N-1]$ and 0 otherwise, then the pulseshaping MC system reduces to a conventional cyclic-prefix OFDM system with cyclic prefix length $N-K \geq 0$.

\section{System Channel}

Combining (1), (5), and (6) yields the following relation between the transmit symbols $a_{l, k}$ and receive symbols $x_{l, k}$ [12]:

$$
x_{l, k}=\sum_{l^{\prime}=-\infty}^{\infty} \sum_{k^{\prime}=0}^{K-1} H_{l, k ; l^{\prime}, k^{\prime}} a_{l^{\prime}, k^{\prime}}+z_{l, k},
$$

where $H_{l, k ; l^{\prime}, k^{\prime}} \triangleq\left\langle\mathbb{H} g_{l^{\prime}, k^{\prime}}, \gamma_{l, k}\right\rangle$ and $z_{l, k} \triangleq\left\langle z, \gamma_{l, k}\right\rangle$. The coefficients $H_{l, k ; l^{\prime}, k^{\prime}}$ for $l, l^{\prime} \in \mathbb{Z}$ and $k, k^{\prime} \in\{0, \ldots, K-1\}$ characterize the system channel comprising the MC modulator, the doubly spread physical channel, and the MC demodulator. In general, this system channel introduces ISI, characterized by $H_{l, k ; l^{\prime}, k^{\prime}}$ for $l \neq l^{\prime}$, and ICI, characterized by $H_{l, k ; l^{\prime}, k^{\prime}}$ for $k \neq k^{\prime}$. Using the cross-ambiguity function $[13,14]$

$$
A_{\gamma, g}(m, \xi) \triangleq \sum_{n=-\infty}^{\infty} \gamma(n) g^{*}(n-m) e^{-j 2 \pi \xi n}
$$

the system channel coefficients can be expressed as

$$
\begin{aligned}
H_{l, k ; l^{\prime}, k^{\prime}}= & \sum_{m=-\infty}^{\infty} \int_{0}^{1} S_{\mathbb{H}}(m, \xi) A_{\gamma_{l, k}, g_{l^{\prime}, k^{\prime}}}^{*}(m, \xi) d \xi \\
= & e^{-j 2 \pi \frac{N}{K} k^{\prime}\left(l^{\prime}-l\right)} \sum_{m=-\infty}^{\infty} \int_{0}^{1} e^{-j 2 \pi\left(m \frac{k^{\prime}}{K}-\xi l N\right)} \\
& \cdot S_{\mathbb{H}}(m, \xi) A_{\gamma, g}^{*}\left(m+\left(l^{\prime}-l\right) N, \xi+\frac{k^{\prime}-k}{K}\right) d \xi,
\end{aligned}
$$

where we have used (2) and the relation $A_{\gamma_{l, k}, g_{l^{\prime}, k^{\prime}}}(m, \xi)=$ $e^{j 2 \pi\left[\frac{N}{K} k^{\prime}\left(l^{\prime}-l\right)+m \frac{k^{\prime}}{K}-\xi l N\right]} A_{\gamma, g}\left(m+\left(l^{\prime}-l\right) N, \xi+\frac{k^{\prime}-k}{K}\right)$.

\section{Statistical ANALYSis}

As a necessary basis for characterizing the CAIC, we next investigate various statistical properties of the MC transmission system including the US doubly spread channel.

\section{A. Statistics of the System Channel}

We first calculate the statistics of $H_{l, k ; l^{\prime}, k^{\prime}}$. Because $H_{l, k ; l^{\prime}, k^{\prime}}$ is zero-mean and rotationally invariant complex Gaussian, it suffices to consider the covariance $\mathrm{E}\left\{H_{l_{1}, k_{1} ; l_{1}^{\prime}, k_{1}^{\prime}} H_{l_{2}, k_{2} ; l_{2}^{\prime}, k_{2}^{\prime}}^{*}\right\}$. However, for reasons that will become clear later on, we consider the covariance of $H_{l, k ; l^{\prime}, k^{\prime}}$ with a cyclic shift by

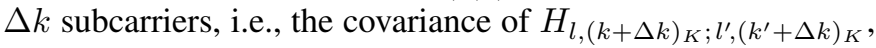
where $(\cdot)_{K}$ is short for modulo $K$. We obtain

$$
\begin{aligned}
& C_{H}^{\Delta k}\left(l_{1}, k_{1} ; l_{1}^{\prime}, k_{1}^{\prime} ; l_{2}, k_{2} ; l_{2}^{\prime}, k_{2}^{\prime}\right) \\
& \triangleq \mathrm{E}\left\{H_{l_{1},\left(k_{1}+\Delta k\right)_{K} ; l_{1}^{\prime},\left(k_{1}^{\prime}+\Delta k\right)_{K}} H_{l_{2},\left(k_{2}+\Delta k\right)_{K} ; l_{2}^{\prime},\left(k_{2}^{\prime}+\Delta k\right)_{K}}^{*}\right\} \\
&= e^{-j 2 \pi \frac{N}{K}\left[\left(k_{1}^{\prime}+\Delta k\right)\left(l_{1}^{\prime}-l_{1}\right)-\left(k_{2}^{\prime}+\Delta k\right)\left(l_{2}^{\prime}-l_{2}\right)\right]} \\
& \cdot \sum_{m=-\infty}^{\infty} \int_{0}^{1} \int_{0}^{1} B_{\mathbb{H}}\left(m, \xi_{1}, \xi_{2}\right) e^{j 2 \pi\left[\left(\xi_{1} l_{1}-\xi_{2} l_{2}\right) N-m \frac{k_{1}^{\prime}-k_{2}^{\prime}}{K}\right]} \\
& \cdot A_{\gamma, g}^{*}\left(m+\left(l_{1}^{\prime}-l_{1}\right) N, \xi_{1}+\frac{k_{1}^{\prime}-k_{1}}{K}\right) \\
& \cdot A_{\gamma, g}\left(m+\left(l_{2}^{\prime}-l_{2}\right) N, \xi_{2}+\frac{k_{2}^{\prime}-k_{2}}{K}\right) d \xi_{1} d \xi_{2},
\end{aligned}
$$

where the relations (7), (4), and $A_{\gamma, g}(m, \xi+1)=A_{\gamma, g}(m, \xi)$ have been used.

Hereafter, we assume that the ISI is negligible, i.e.,

$$
H_{l, k ; l^{\prime}, k^{\prime}}=0 \quad \text { for } l \neq l^{\prime} .
$$

Indeed, in the important special case of cyclic-prefix OFDM, the ISI completely vanishes provided that the channel's maximum delay is below the length of the cyclic prefix. More generally, it is shown in [15] that there exist perfect-reconstruction pulses $g$ and $\gamma$ that are jointly well time-frequency localized, i.e., $A_{g, g}(m, \xi), A_{\gamma, \gamma}(m, \xi)$, and $A_{\gamma, g}(m, \xi)$ are fast decaying, yielding MC systems with almost vanishing ISI. We note that our analysis remains valid even for strong ICI, i.e., for rapidly time-varying channels. This is a difference from other approaches which assume that both ISI and ICI are negligible (e.g., [16]; note, however, that [16] does not assume that channel state information is available at the receiver).

Negligible ISI means that $C_{H}^{\Delta k}\left(l_{1}, k_{1} ; l_{1}^{\prime}, k_{1}^{\prime} ; l_{2}, k_{2} ; l_{2}^{\prime}, k_{2}^{\prime}\right) \approx$ 0 for $l_{1} \neq l_{1}^{\prime}$ or $l_{2} \neq l_{2}^{\prime}$, and therefore it suffices to consider the case $l_{1}=l_{1}^{\prime}$ and $l_{2}=l_{2}^{\prime}$. Here, (8) simplifies to

$$
\begin{aligned}
& C_{H}^{\Delta k}\left(l_{1}, k_{1} ; l_{1}, k_{1}^{\prime} ; l_{2}, k_{2} ; l_{2}, k_{2}^{\prime}\right) \\
& =\sum_{m=-\infty}^{\infty} \int_{0}^{1} \int_{0}^{1} B_{\mathbb{H}}\left(m, \xi_{1}, \xi_{2}\right) e^{j 2 \pi\left[\left(\xi_{1} l_{1}-\xi_{2} l_{2}\right) N-m \frac{k_{1}^{\prime}-k_{2}^{\prime}}{K}\right]} \\
& \quad \cdot A_{\gamma, g}^{*}\left(m, \xi_{1}+\frac{k_{1}^{\prime}-k_{1}}{K}\right) A_{\gamma, g}\left(m, \xi_{2}+\frac{k_{2}^{\prime}-k_{2}}{K}\right) d \xi_{1} d \xi_{2},
\end{aligned}
$$

which does not depend on $\Delta k$. Hence, with our assumption of negligible ISI, the statistics of the system channel are invariant under cyclic shifts of the subcarriers. 


\section{B. Mean Transmit Power}

Next, we calculate the mean power of the transmit signal $s(n)$ in (5) averaged over a symbol period of length $N$. Let

$$
C_{a}\left(l_{1}, k_{1} ; l_{2}, k_{2}\right) \triangleq \mathrm{E}\left\{a_{l_{1}, k_{1}} a_{l_{2}, k_{2}}^{*}\right\}
$$

denote the covariance of the (zero-mean) data symbols $a_{l, k}$. As before, we assume a pulse design that guarantees (almost) ISIfree transmission. More specifically, we assume that the transmit pulse $g(n)$ is supported in $[0, N-1]$, so that $A_{g, g}(m, \xi)$ $=0$ for $m$ outside $[-N+1, N-1]$. We then obtain the mean transmit power in the $l$ th symbol interval as

$$
\begin{aligned}
P_{s}(l) & \triangleq \frac{1}{N} \sum_{n=0}^{N-1} \mathrm{E}\left\{|s(l N+n)|^{2}\right\} \\
& =\frac{1}{N} \sum_{k_{1}=0}^{K-1} \sum_{k_{2}=0}^{K-1} C_{a}\left(l, k_{1} ; l, k_{2}\right) \sum_{n=0}^{N-1} g_{0, k_{1}}(n) g_{0, k_{2}}^{*}(n) \\
& =\frac{1}{N} \sum_{k_{1}=0}^{K-1} \sum_{k_{2}=0}^{K-1} C_{a}\left(l, k_{1} ; l, k_{2}\right) A_{g, g}\left(0, \frac{k_{2}-k_{1}}{K}\right) .
\end{aligned}
$$

For a cyclic shift of the data symbols $a_{l, k}$ by $\Delta k$ subcarriers, we obtain

$$
\begin{array}{r}
P_{s}^{\Delta k}(l)=\frac{1}{N} \sum_{k_{1}=0}^{K-1} \sum_{k_{2}=0}^{K-1} C_{a}\left(l,\left(k_{1}+\Delta k\right)_{K} ; l,\left(k_{2}+\Delta k\right)_{K}\right) \\
\cdot A_{g, g}\left(0, \frac{k_{2}-k_{1}}{K}\right),
\end{array}
$$

which is easily seen to be equal to $P_{s}(l)$. Thus, the mean transmit power is invariant under cyclic shifts of the subcarriers.

\section{Noise Statistics}

Finally, we calculate the statistics of the noise samples $z_{l, k}=\left\langle z, \gamma_{l, k}\right\rangle$ at the output of the system channel. Due to the assumptions on $z(n)$ made in Section II-A, the $z_{l, k}$ 's are zeromean, rotationally invariant, jointly complex Gaussian with covariance $C_{z}\left(l_{1}, k_{1} ; l_{2}, k_{2}\right) \triangleq \mathrm{E}\left\{z_{l_{1}, k_{1}} z_{l_{2}, k_{2}}^{*}\right\}$ given by

$$
\begin{aligned}
C_{z}\left(l_{1}, k_{1} ; l_{2}, k_{2}\right) & =\sigma_{z}^{2} \sum_{n=-\infty}^{\infty} \gamma_{l_{1}, k_{1}}^{*}(n) \gamma_{l_{2}, k_{2}}(n) \\
& =\sigma_{z}^{2} e^{j 2 \pi \frac{N}{K} k_{1}\left(l_{1}-l_{2}\right)} A_{\gamma, \gamma}\left(\left(l_{1}-l_{2}\right) N, \frac{k_{1}-k_{2}}{K}\right) .
\end{aligned}
$$

We again assume (almost) ISI-free transmission-more specifically, that $A_{\gamma, \gamma}(m, \xi)=0$ is zero for $m$ outside $[-N+$ $1, N-1]$. Then $C_{z}\left(l_{1}, k_{1} ; l_{2}, k_{2}\right)=0$ for $l_{1} \neq l_{2}$, which shows that noise samples $z_{l, k}$ for different $l$ are uncorrelated. It thus suffices to consider the case $l_{1}=l_{2}=l$, where

$$
C_{z}\left(l, k_{1} ; l, k_{2}\right)=\sigma_{z}^{2} A_{\gamma, \gamma}\left(0, \frac{k_{1}-k_{2}}{K}\right) .
$$

From this expression, it is seen that the noise at the output of the system channel is stationary and its statistics are invariant under cyclic shifts of the subcarriers. We note that the same statistical structure would be obtained with an orthogonal [15] receive pulse even if it is not well concentrated.

\section{CAPACITY-ACHIEVIng InPut Covariance}

\section{A. System Capacity}

We will now consider the capacity of the system channel consisting of the MC modulator, the US doubly selective channel, and the MC demodulator. Let $L$ be a given time horizon. We introduce the nonnegative definite input covariance matrix of size $(2 L+1) K \times(2 L+1) K$

$$
\mathbf{C}_{a} \triangleq\left[\begin{array}{ccc}
\mathbf{C}_{a}^{(-L,-L)} & \cdots & \mathbf{C}_{a}^{(-L, L)} \\
\vdots & \ddots & \vdots \\
\mathbf{C}_{a}^{(L,-L)} & \cdots & \mathbf{C}_{a}^{(L, L)}
\end{array}\right]
$$

with the matrix blocks $\mathbf{C}_{a}^{\left(l_{1}, l_{2}\right)}$ of size $K \times K$ given by

$$
\left(\mathbf{C}_{a}^{\left(l_{1}, l_{2}\right)}\right)_{k_{1}, k_{2}} \triangleq C_{a}\left(l_{1}, k_{1} ; l_{2}, k_{2}\right), \quad k_{1}, k_{2}=0, \ldots, K-1 .
$$

In a similar way, we define the $(2 L+1) K \times(2 L+1) K$ channel matrix $\mathbf{H}$ and noise covariance matrix $\mathbf{C}_{z}$ with $K \times K$ blocks $\mathbf{H}^{\left(l_{1}, l_{2}\right)}$ and $\mathbf{C}_{z}^{\left(l_{1}, l_{2}\right)}$ given by $\left(\mathbf{H}^{\left(l_{1}, l_{2}\right)}\right)_{k_{1}, k_{2}} \triangleq H_{l_{1}, k_{1} ; l_{2}, k_{2}}$ and $\left(\mathbf{C}_{z}^{\left(l_{1}, l_{2}\right)}\right)_{k_{1}, k_{2}} \triangleq C_{z}\left(l_{1}, k_{1} ; l_{2}, k_{2}\right)$, respectively. Due to our assumption of ISI-free transmission and the results of the previous section, $\mathbf{H}$ and $\mathbf{C}_{z}$ are block-diagonal, i.e.,

$$
\begin{aligned}
\mathbf{H} & =\operatorname{diag}\left\{\mathbf{H}^{(-L,-L)}, \ldots, \mathbf{H}^{(L, L)}\right\} \\
\mathbf{C}_{z} & =\operatorname{diag}\left\{\mathbf{C}_{z}^{(-L,-L)}, \ldots, \mathbf{C}_{z}^{(L, L)}\right\} .
\end{aligned}
$$

If instantaneous channel state information is available at the receiver but not at the transmitter, and if the channel statistics are known at both sides, the (ergodic) capacity of the system channel is given by (cf. [17]; see also [1,2])

with

$$
C=\lim _{L \rightarrow \infty} \frac{1}{2 L+1} \max _{\mathbf{C}_{a} \in \mathcal{M}_{a}} \mathrm{E}\left\{\Psi\left(\mathbf{C}_{a}, \mathbf{H}, \mathbf{C}_{z}\right)\right\}
$$

$$
\Psi\left(\mathbf{C}_{a}, \mathbf{H}, \mathbf{C}_{z}\right) \triangleq \log \operatorname{det}\left(\mathbf{H} \mathbf{C}_{a} \mathbf{H}^{H}+\mathbf{C}_{z}\right)-\log \operatorname{det}\left(\mathbf{C}_{z}\right),
$$

where it is assumed that the limit in (10) exists. Note that $\mathrm{E}\left\{\Psi\left(\mathbf{C}_{a}, \mathbf{H}, \mathbf{C}_{z}\right)\right\}$ depends on $L$ through the dimension of the matrices involved. The expectation in (10) is with respect to $\mathbf{H}$. The maximization is over the set $\mathcal{M}_{a}$ of all input covariance matrices $\mathbf{C}_{a}$ such that the mean transmit power function $P_{s}(l), l \in \mathbb{Z}$ at the input of the physical channel satisfies a given constraint (whose exact nature is irrelevant to our analysis). We recall that $P_{s}(l)$ depends on $\mathbf{C}_{a}$ via (9), which can be written as $P_{s}(l)=\frac{1}{N} \operatorname{tr}\left\{\mathbf{C}_{a}^{(l, l)} \mathbf{A}_{g}^{T}\right\}$ where $\left(\mathbf{A}_{g}\right)_{k_{1}, k_{2}} \triangleq A_{g, g}\left(0, \frac{k_{2}-k_{1}}{K}\right)$ and $\operatorname{tr}$ denotes the trace. In contrast to classical OFDM systems where ICI is considered a parasitic effect, our model makes use of all "cross-channels" established by the ICI; these are represented by the offdiagonal elements of the channel matrices $\mathbf{H}^{(l, l)}$.

\section{B. Block-Diagonal Structure}

We shall now derive structural properties of the optimum input covariance matrix (CAIC) $\mathbf{C}_{a}$, i.e., of the $\mathbf{C}_{a}$ maximizing $\mathrm{E}\left\{\Psi\left(\mathbf{C}_{a}, \mathbf{H}, \mathbf{C}_{z}\right)\right\}$ in (10). First, consider some input 
covariance matrix $\mathbf{C}_{a}$ and its block-diagonal version in which all off-diagonal matrix blocks are zero,

$$
\widetilde{\mathbf{C}}_{a} \triangleq \operatorname{diag}\left\{\mathbf{C}_{a}^{(-L,-L)}, \ldots, \mathbf{C}_{a}^{(L, L)}\right\} .
$$

Note that $\widetilde{\mathbf{C}}_{a}$ corresponds to data symbols $a_{l, k}$ that are uncorrelated over time $(l)$. Because $P_{s}(l)$ in (9) depends only on the diagonal blocks $\mathbf{C}_{a}^{(l, l)}$, it is clear that $\widetilde{\mathbf{C}}_{a}$ satisfies the power constraint if and only if $\mathbf{C}_{a}$ does. Furthermore, consider the covariance matrix of the system channel output $x_{k, l}$

$$
\mathbf{C}_{x}=\mathbf{H C}_{a} \mathbf{H}^{H}+\mathbf{C}_{z}=\left[\begin{array}{ccc}
\mathbf{C}_{x}^{(-L,-L)} & \cdots & \mathbf{C}_{x}^{(-L, L)} \\
\vdots & \ddots & \vdots \\
\mathbf{C}_{x}^{(L,-L)} & \cdots & \mathbf{C}_{x}^{(L, L)}
\end{array}\right]
$$

and note that (11) can be written as

$$
\Psi\left(\mathbf{C}_{a}, \mathbf{H}, \mathbf{C}_{z}\right)=\log \operatorname{det}\left(\mathbf{C}_{x}\right)-\log \operatorname{det}\left(\mathbf{C}_{z}\right) .
$$

Let $\widetilde{\mathbf{C}}_{x} \triangleq \operatorname{diag}\left\{\mathbf{C}_{x}^{(-L,-L)}, \ldots, \mathbf{C}_{x}^{(L, L)}\right\}$ be the block-diagonal version of $\mathbf{C}_{x}$. We have $\widetilde{\mathbf{C}}_{x}=\mathbf{H} \widetilde{\mathbf{C}}_{a} \mathbf{H}^{H}+\mathbf{C}_{z}$, i.e., $\widetilde{\mathbf{C}}_{x}$ is obtained when $\mathbf{C}_{a}$ is replaced by $\widetilde{\mathbf{C}}_{a}$. Thus, in particular,

$$
\Psi\left(\widetilde{\mathbf{C}}_{a}, \mathbf{H}, \mathbf{C}_{z}\right)=\log \operatorname{det}\left(\widetilde{\mathbf{C}}_{x}\right)-\log \operatorname{det}\left(\mathbf{C}_{z}\right) .
$$

From Hadamard's inequality ${ }^{1} \operatorname{det}\left(\mathbf{C}_{x}\right) \leq \operatorname{det}\left(\widetilde{\mathbf{C}}_{x}\right)$, it follows with (12) and (13) that $\Psi\left(\mathbf{C}_{a}, \mathbf{H}, \mathbf{C}_{z}\right) \leq \Psi\left(\widetilde{\mathbf{C}}_{a}, \mathbf{H}, \mathbf{C}_{z}\right)$ for any given $\mathbf{H}$ and, thus,

$$
\mathrm{E}\left\{\Psi\left(\widetilde{\mathbf{C}}_{a}, \mathbf{H}, \mathbf{C}_{z}\right)\right\} \geq \mathrm{E}\left\{\Psi\left(\mathbf{C}_{a}, \mathbf{H}, \mathbf{C}_{z}\right)\right\} .
$$

We conclude that for any $\mathbf{C}_{a}$ satisfying the power constraint, we always find a block-diagonal $\widetilde{\mathbf{C}}_{a}$ that also satisfies the power constraint and is at least as good (in the sense of (14)) as $\mathbf{C}_{a}$. This holds for all $L$. Therefore, we can restrict the set $\mathcal{M}_{a}$ of admissible input covariance matrices $\mathbf{C}_{a}$ in (10) to the set of all block-diagonal covariance matrices.

\section{Circulant Structure}

We shall derive a further structural property of the optimum $\mathbf{C}_{a}$. Let $\mathbf{a}_{l} \triangleq\left[a_{l, 0} \cdots a_{l, K-1}\right]^{T}$ be the $l$ th symbol vector, and let the $K \times K$ matrix $\mathbf{S}_{\Delta k}$ perform a cyclic shift by $\Delta k$ positions. When the data symbols $a_{l, k}$ are cyclically shifted by $\Delta k$ subcarriers, i.e., $\mathbf{a}_{l} \mapsto \mathbf{S}_{\Delta k} \mathbf{a}_{l}=\left[a_{l,(\Delta k)_{K}}\right.$ $\left.\cdots a_{l,(K-1+\Delta k)_{K}}\right]^{T}$, the input covariance matrix (which is assumed block-diagonal as discussed above) becomes

$$
\mathbf{C}_{a}^{\Delta k} \triangleq \mathbf{T}_{\Delta k} \mathbf{C}_{a} \mathbf{T}_{\Delta k}^{T},
$$

with the block-diagonal $(2 L+1) K \times(2 L+1) K$ matrix $\mathbf{T}_{\Delta k}$ $\triangleq \operatorname{diag}\left\{\mathbf{S}_{\Delta k}, \ldots, \mathbf{S}_{\Delta k}\right\}$. Note that $\mathbf{C}_{a}^{\Delta k}$ is again block-diagonal. According to Section III-B, $P_{s}(l)$ is invariant under cyclic shifts of the subcarriers, and thus $\mathbf{C}_{a}^{\Delta k}$ satisfies the power constraint if and only if $\mathbf{C}_{a}$ does. Furthermore, consider

$$
\begin{aligned}
& \Psi\left(\mathbf{C}_{a}^{\Delta k}, \mathbf{H}, \mathbf{C}_{z}\right) \\
& \quad=\log \operatorname{det}\left(\mathbf{H T}_{\Delta k} \mathbf{C}_{a} \mathbf{T}_{\Delta k}^{T} \mathbf{H}^{H}+\mathbf{C}_{z}\right)-\log \operatorname{det}\left(\mathbf{C}_{z}\right) .
\end{aligned}
$$

\footnotetext{
${ }^{1}$ This block version of Hadamard's inequality can be shown by extending the derivation in $[18$, p. 680], that is, by recursive application of the bound on differential entropy $h\left(X_{1}, \ldots, X_{m}, X_{m+1}, \ldots, X_{n}\right) \leq h\left(X_{1}, \ldots, X_{m}\right)+$ $h\left(X_{m+1}, \ldots, X_{n}\right)$ to jointly Gaussian random variables $X_{1}, \ldots, X_{n}$.
}

Using the fact that $\operatorname{det}(\mathbf{C})=\operatorname{det}\left(\mathbf{T}_{\Delta k}^{T} \mathbf{C} \mathbf{T}_{\Delta k}\right)$ for any $(2 L+$ 1) $K \times(2 L+1) K$ matrix $\mathbf{C}$, it is straightforward to show that

$$
\Psi\left(\mathbf{C}_{a}^{\Delta k}, \mathbf{H}, \mathbf{C}_{z}\right)=\Psi\left(\mathbf{C}_{a}, \mathbf{T}_{\Delta k}^{T} \mathbf{H T}_{\Delta k}, \mathbf{T}_{\Delta k}^{T} \mathbf{C}_{z} \mathbf{T}_{\Delta k}\right) .
$$

As was demonstrated in Section III-C, the statistics of the output noise $z_{l, k}$ are invariant under cyclic shifts of the subcarriers. It then follows that $\mathbf{T}_{\Delta k}^{T} \mathbf{C}_{z} \mathbf{T}_{\Delta k}=\mathbf{C}_{z}$, and hence (15) becomes

$$
\Psi\left(\mathbf{C}_{a}^{\Delta k}, \mathbf{H}, \mathbf{C}_{z}\right)=\Psi\left(\mathbf{C}_{a}, \mathbf{T}_{\Delta k}^{T} \mathbf{H} \mathbf{T}_{\Delta k}, \mathbf{C}_{z}\right) .
$$

Furthermore, we have shown in Section III-A that the statistics of $H_{l, k ; l^{\prime}, k^{\prime}}$ are invariant under cyclic shifts of the subcarriers. Hence, the statistics of $\mathbf{T}_{\Delta k}^{T} \mathbf{H} \mathbf{T}_{\Delta k}$ equal those of $\mathbf{H}$, which implies that $\mathrm{E}\left\{\Psi\left(\mathbf{C}_{a}, \mathbf{T}_{\Delta k}^{T} \mathbf{H T}_{\Delta k}, \mathbf{C}_{z}\right)\right\}=$ $\mathrm{E}\left\{\Psi\left(\mathbf{C}_{a}, \mathbf{H}, \mathbf{C}_{z}\right)\right\}$. From (16), we then obtain

$$
\mathrm{E}\left\{\Psi\left(\mathbf{C}_{a}^{\Delta k}, \mathbf{H}, \mathbf{C}_{z}\right)\right\}=\mathrm{E}\left\{\Psi\left(\mathbf{C}_{a}, \mathbf{H}, \mathbf{C}_{z}\right)\right\} .
$$

We conclude that if $\mathbf{C}_{a}$ maximizes $\mathrm{E}\left\{\Psi\left(\mathbf{C}_{a}, \mathbf{H}, \mathbf{C}_{z}\right)\right\}$, then so does $\mathbf{C}_{a}^{\Delta k}$. Thus, the optimum input covariance $\mathbf{C}_{a}$ is only defined up to an arbitrary cyclic shift of the subcarriers.

Let us next consider the average of all matrices $\mathbf{C}_{a}^{\Delta k}=$ $\mathbf{T}_{\Delta k} \mathbf{C}_{a} \mathbf{T}_{\Delta k}^{T}$

$$
\hat{\mathbf{C}}_{a} \triangleq \frac{1}{K} \sum_{\Delta k=0}^{K-1} \mathbf{C}_{a}^{\Delta k} \text {. }
$$

This is again a block-diagonal matrix. Furthermore, due to the construction (17), the diagonal blocks $\hat{\mathbf{C}}_{a}^{(l, l)}$ of $\hat{\mathbf{C}}_{a}$ are circulant matrices. Because $P_{s}(l)$ is linear in $\mathbf{C}_{a}$ and invariant under cyclic subcarrier shifts, $\hat{\mathbf{C}}_{a}$ satisfies the power constraint if and only if $\mathbf{C}_{a}$ does. Moreover, due to the concavity of the mapping $\mathbf{C}_{a} \mapsto \mathrm{E}\left\{\Psi\left(\mathbf{C}_{a}, \mathbf{H}, \mathbf{C}_{z}\right)\right\}$ [17], we have

$$
\mathrm{E}\left\{\Psi\left(\hat{\mathbf{C}}_{a}, \mathbf{H}, \mathbf{C}_{z}\right)\right\} \geq \mathrm{E}\left\{\Psi\left(\mathbf{C}_{a}, \mathbf{H}, \mathbf{C}_{z}\right)\right\} .
$$

Thus, for any $\mathbf{C}_{a}$ satisfying the power constraint, we always find a $\hat{\mathbf{C}}_{a}$ that is block-diagonal with circulant diagonal blocks, that also satisfies the power constraint, and that is at least as good (in the sense of (18)) as $\mathbf{C}_{a}$. This holds for all $L$, and hence also in the limit $L \rightarrow \infty$.

We have thus shown the following structural result: there always is a CAIC of the form

$$
\hat{C}_{a}\left(l_{1}, k_{1} ; l_{2}, k_{2}\right)=\delta\left(l_{1}-l_{2}\right) c_{a}^{\left(l_{1}\right)}\left(\left(k_{1}-k_{2}\right)_{K}\right),
$$

with some $l$-dependent covariance function $c_{a}^{(l)}(\kappa)$ that remains to be optimized in the sense of (10). The form (19) corresponds to transmit symbols $a_{l, k}$ that are uncorrelated over time $(l)$ and "cyclically stationary" with respect to $k$.

\section{Capacity-Achieving Precoding And PULSE DESIGN}

Finally, we discuss ways of realizing the CAIC (19). The transmit signal in (5) can be written as $s(n)=\sum_{l=-\infty}^{\infty} s_{l}(n)$ with

$$
\begin{aligned}
s_{l}(n) & =\sum_{k=0}^{K-1} a_{l, k} g(n-l N) e^{j 2 \pi \frac{k}{K}(n-l N)} \\
& =\sqrt{K}\left(\mathbf{g} \odot\left(\mathbf{Q F}^{H} \mathbf{a}_{l}\right)\right)_{n-l N},
\end{aligned}
$$


where $\left(\mathbf{a}_{l}\right)_{k}=a_{l, k}$ and $(\mathbf{g})_{n}=g(n) ; \mathbf{F}$ is the $K \times K$ discrete Fourier transform (DFT) matrix, i.e., $(\mathbf{F})_{k, n}=$ $e^{-j 2 \pi n k / K} / \sqrt{K} ; \mathbf{Q}$ performs a $K$-periodic extension of a $K$-dimensional vector; and $\odot$ denotes elementwise vector multiplication.

We have shown in the previous section that the capacityachieving covariance matrices $\hat{\mathbf{C}}_{a}^{(l, l)}$ are circulant matrices. They can thus be diagonalized by the DFT, that is,

$$
\hat{\mathbf{C}}_{a}^{(l, l)}=\mathbf{F D}_{l} \mathbf{F}^{H},
$$

where $\mathbf{D}_{l}$ is the diagonal eigenvalue matrix. The CAIC can then be realized by using uncorrelated, unit-variance data symbols $\tilde{a}_{l, k}$, from which the symbol vectors $\mathbf{a}_{l}$ used in (20) are derived as

$$
\mathbf{a}_{l}=\mathbf{P}_{l} \tilde{\mathbf{a}}_{l},
$$

with the precoder matrices

$$
\mathbf{P}_{l} \triangleq \mathbf{F D}_{l}^{1 / 2} \mathbf{F}^{H} .
$$

Here, $\mathbf{D}_{l}^{1 / 2}$ is the diagonal matrix containing the square roots of the eigenvalues of $\hat{\mathbf{C}}_{a}^{(l, l)}$. Note that $\mathbf{P}_{l} \mathbf{P}_{l}^{H}=\hat{\mathbf{C}}_{a}^{(l, l)}$.

We will now derive an interesting "pulse design" representation of the precoding operation. Inserting (21) into (20) and using (22) as well as $\mathbf{F}^{H} \mathbf{F}=\mathbf{I}$ yields

$$
s_{l}(n)=\sqrt{K}\left(\mathbf{g} \odot\left(\mathbf{Q D}_{l}^{1 / 2} \mathbf{F}^{H} \tilde{\mathbf{a}}_{l}\right)\right)_{n-l N} .
$$

This can be rewritten as

$$
s_{l}(n)=\sqrt{K}\left(\tilde{\mathbf{g}}_{l} \odot\left(\mathbf{Q F}^{H} \tilde{\mathbf{a}}_{l}\right)\right)_{n-l N},
$$

with

$$
\tilde{\mathbf{g}}_{l} \triangleq \mathbf{g} \odot\left(\mathbf{Q} \mathbf{d}_{l}\right),
$$

where the vector $\mathbf{d}_{l}$ is the diagonal of $\mathbf{D}_{l}^{1 / 2}$, consisting of the square roots of the eigenvalues of $\hat{\mathbf{C}}_{a}^{(l, l)}$. We observe that (23) is of the form (20), except that $\mathbf{g}$ and $\mathbf{a}_{l}$ are replaced by $\tilde{\mathbf{g}}_{l}$ and $\tilde{\mathbf{a}}_{l}$, respectively. This shows that the capacityachieving precoding can be equivalently realized by a timevarying adaptation of the transmit pulse $\mathrm{g}$ according to (24).

If the physical channel is WSSUS, its statistics are stationary and thus $\hat{\mathbf{C}}_{a}^{(l, l)}$ is no longer time-dependent. The same then holds for the adapted transmit pulse, i.e., $\tilde{\mathbf{g}}_{l} \equiv \tilde{\mathbf{g}}$. Hence, capacity-achieving precoding can be realized by a simple "capacity-achieving pulse design." For classical cyclic-prefix OFDM, this means that a precoding can be avoided if pulseshaping MC transmission is used instead. We note that pulseshaping MC systems can be implemented efficiently by means of fast Fourier transform and overlap-add techniques [15].

\section{CONCLUSION}

We considered pulse-shaping multicarrier (MC) communications over a doubly selective fading channel with uncorrelated scattering. Classical cyclic-prefix OFDM systems and WSSUS channels are special cases of our setting. A pulse design avoiding intersymbol interference was assumed; however, intercarrier interference was not restricted, thus allowing for rapidly time-varying channels. We showed that the transfer and noise statistics of the system channel are uncorrelated over time, and that the mean transmit power at the input of the physical channel as well as the transfer and noise statistics of the system channel are invariant under cyclic shifts of the subcarriers. This statistical structure was then shown to entail a characteristic structure of the capacity-achieving input covariance, corresponding to input symbols that are uncorrelated over time and cyclically stationary with respect to the frequency (subcarrier) index. We also showed that this statistical structure can be realized by an adaptation of the transmit pulse that is time-invariant for a WSSUS channel. For classical OFDM, in particular, precoding can be completely avoided if pulse-shaping MC transmission is used instead.

\section{ACKNOWLEDGMENT}

This work was supported by the WWTF under grant MA 44 (MOHAWI).

\section{REFERENCES}

[1] Ke Liu, T. Kadous, and A. M. Sayeed, "Orthogonal time-frequency signaling over doubly dispersive channels," IEEE Trans. Inf. Theory, vol. 50, pp. 2583-2603, Nov. 2004.

[2] V. Kafedziski, "Capacity of frequency selective fading channels with side information," in Proc. 32th Asilomar Conf. Signals, Systems, Computers, vol. 2, (Pacific Grove, CA), pp. 1758-1762, Nov. 1998.

[3] A. Szabo, T. P. Kurpjuhn, and W. Utschick, "Linearly prefiltered OFDM based on long-term properties of the channel covariance matrix," in Proc. IEEE VTC-2002 (fall), vol. 4, (Vancouver, Canada), pp. 2066-2070, Sept. 2002.

[4] Zhengdao Wang and G. B. Giannakis, "Complex-field coding for OFDM over fading wireless channels," IEEE Trans. Inf. Theory, vol. 49, pp. 707-720, March 2003.

[5] Yue Rong, S. A. Vorobyov, and A. B. Gershman, "Linear block precoding for OFDM systems based on maximization of mean cutoff rate," IEEE Trans. Signal Processing, vol. 53, pp. 4691-4696, Dec. 2005.

[6] P. A. Bello, "Characterization of randomly time-variant linear channels," IEEE Trans. Comm. Syst., vol. 11, pp. 360-393, 1963.

[7] A. M. Tulino, A. Lozano, and S. Verdu, "Capacity-achieving input covariance for single-user multi-antenna channels," IEEE Trans. Wireless Comm., vol. 5, pp. 662-671, March 2006.

[8] I. Bjelakovic and H. Boche, "Structure of optimal input covariance matrices for MIMO systems with covariance feedback under general correlated fading," in Proc. IEEE ISIT-06, (Seattle, WA), pp. 1041-1045, July 2006.

[9] W. Rhee and G. Taricco, "On the ergodic capacity-achieving covariance matrix of certain classes of MIMO channels," IEEE Trans. Inf. Theory, vol. 52, pp. 3810-3817, Aug. 2006.

[10] L. W. Hanlen and A. J. Grant, "Optimal transmit covariance for MIMO channels with statistical transmitter side information," in Proc. IEEE ISIT-05, (Adelaide, Australia), pp. 1818-1822, Sept. 2005.

[11] E. Abbe, E. Telatar, and L. Zheng, "The algebra of MIMO channels," in Proc. 43rd Allerton Conf. Commun., Contr., Comput., (Urbana, IL), Oct. 2005.

[12] W. Kozek and A. F. Molisch, "Nonorthogonal pulseshapes for multicarrier communications in doubly dispersive channels," IEEE J. Sel. Areas Comm., vol. 16, pp. 1579-1589, Oct. 1998.

[13] P. Flandrin, Time-Frequency/Time-Scale Analysis. San Diego (CA): Academic Press, 1999.

[14] K. Gröchenig, Foundations of Time-Frequency Analysis. Boston (MA): Birkhäuser, 2001.

[15] G. Matz, D. Schafhuber, K. Gröchenig, M. Hartmann, and F. Hlawatsch, "Analysis, optimization, and implementation of low-interference wireless multicarrier systems," to appear in IEEE Trans. Wireless Comm., 2007.

[16] G. Durisi, H. Bölcskei, and S. Shamai (Shitz), "Capacity of underspread WSSUS fading channels in the wideband regime," in Proc. IEEE ISIT06, (Seattle, WA), pp. 1500-1504, July 2006.

[17] I. E. Telatar, "Capacity of multi-antenna Gaussian channels," Europ. Trans. Telecomm., vol. 10, pp. 585-595, Nov./Dec. 1999.

[18] T. M. Cover and J. A. Thomas, Elements of Information Theory. New York: Wiley, 2nd ed., 2006 